\title{
Allele Frequency Distribution of the NCAPG c.1326T>G SNP Associated with Growth-Related Traits in Niigata Population of Japanese Black Beef Cattle
}

\author{
Bin Tong1, Youji Muramatsu², Narumi Fuke1, Yui Himizu1, Hiroyuki Katou3, Takeshi Ohta4, \\ Hiroyuki Kose ${ }^{5}$, Takahisa Yamada ${ }^{*}$ \\ ${ }^{1}$ Laboratory of Animal Genetics, Graduate School of Science and Technology, Niigata University, Niigata, Japan \\ ${ }^{2}$ Department of Nutritional Sciences for Well-Being, Faculty of Health Sciences for Welfare, Kansai University of \\ Welfare Sciences, Osaka, Japan \\ ${ }^{3}$ Niigata Prefectural Headquarters, National Federation of Agricultural Cooperative Association, Niigata, Japan \\ ${ }^{4}$ Central Pharmaceutical Research Institute, Japan Tobacco, Inc., Osaka, Japan \\ ${ }^{5}$ Department of Life Science, Division of Natural Sciences, International Christian University, Tokyo, Japan \\ Email: *tyamada@agr.niigata-u.ac.jp
}

Received 28 May 2016; accepted 27 June 2016; published 30 June 2016

Copyright (C) 2016 by authors and Scientific Research Publishing Inc.

This work is licensed under the Creative Commons Attribution International License (CC BY).

http://creativecommons.org/licenses/by/4.0/

(c) (i) Open Access

\section{Abstract}

Growth performance as well as marbling is the main breeding objectives in Japanese Black cattle, the major beef breed in Japan. A previous study has identified the $c .1326 T>G$ single nucleotide polymorphism (SNP) in the NCAPG (non-SMC condensin I complex, subunit G) gene that leads to the amino acid change p.Ile442Met in the NCAPG protein, which is a candidate causative variation for a bovine carcass weight (CWT) quantitative trait locus (QTL). In this study, we first confirmed the association of the $c .1326 T>G$ SNP with the growth-related traits, CWT, rib eye area (REA) and rib thickness (RT), and showed significant effect of the SNP genotypes on the marbling trait, beef marbling score (BMS), in the Japanese Black beef cattle population of the Niigata prefecture, with the $G$ allele being associated with a favorable phenotype of these traits. Thus, we concluded that the $c .1326 T>G$ SNP is useful for effective marker-assisted selection to increase meat quality and meat productivity in Japanese Black beef cattle of the Niigata prefecture. Furthermore the frequency of the favorable G allele of the $c .1326 T>G$ SNP in the Niigata prefecture population was significantly lower than the frequency of this allele in Japanese Black cattle population of the Kagoshima prefecture. However, no statistically significant difference was detected between the allele frequencies estimated by maternal alleles in the half-sib progeny steers in the Niigata prefecture population and obtained in Japanese Black cattle population of the Kagoshima prefecture.

${ }^{*}$ Corresponding author.

How to cite this paper: Tong, B., Muramatsu, Y., Fuke, N., Himizu, Y., Katou, H., Ohta, T., Kose, H. and Yamada, T. (2016) Allele Frequency Distribution of the NCAPG c.1326T>G SNP Associated with Growth-Related Traits in Niigata Population of Japanese Black Beef Cattle. Open Journal of Animal Sciences, 6, 202-206. http://dx.doi.org/10.4236/ojas.2016.63026 


\section{Keywords}

\section{Allele Frequency, Growth-Related Traits, Japanese Black Breed, NCAPG, Single Nucleotide Polymorphism}

\section{Introduction}

Growth performance has an important effect on the economics of beef production [1]. Thus, it is greatly interesting to obtain better knowledge on the molecular architecture of growth characteristics and to generate new opportunities for more effective marker-assisted selection.

A previous study showed that the $c .1326 T>G$ single nucleotide polymorphism (SNP) in the NCAPG (non$S M C$ condensin I complex, subunit $G$ ) gene, which leads to the amino acid change of Ile442 to Met442 in the encoded protein, was identified as a candidate causative variation for a bovine carcass weight (CWT) quantitative trait locus (QTL) on bovine chromosome 6 [2]. The NCAPG c.1326T>G variation is present in various bovine breeds and has linkage or association with birth weight [3] [4], and withers height and body weight at adolescence [4]-[6]. Recently, Hoshiba et al. [7] showed that the c.1326T>G SNP was associated with CWT and rib eye area (REA), subcutaneous fat thickness (SFT) and carcass yield estimate (YE) in Japanese Black beef cattle population in the Kagoshima prefecture, with the $G$ allele of the SNP being associated with a high level of the CWT, REA and YE, and a low level of the SFT.

Thus, it was necessary to investigate whether the association of the $c .1326 T>G$ SNP with growth-related traits could be replicated in Japanese Black beef cattle population of the Niigata prefecture and to analyze the effects of the SNP genotypes on the meat quality trait, beef marbling score (BMS), in order to confirm the application of the $c .1326 T>G$ SNP to effective marker-assisted selection in the Niigata population of Japanese Black cattle. Additionally, we investigated allele frequency distribution of the SNP in the Niigata population.

\section{Materials and Methods}

\subsection{Samples and Data}

We used independent Japanese Black cattle population of the Niigata prefecture, and studied the association of the $c .1326 T>G$ SNP with the BMS, CWT, REA, rib thickness (RT), YE and SFT. In this study, 149 paternal half-sib progeny steers (1 to 16 steers per sire) produced from 39 sires were used. There was no strong bias for a specific father or a specific maternal grandfather of the sires and the sire panel likely represents a variety of the sire lines, indicating that the number of the progeny steers is sufficient for the analyses of this study. Hair root specimens of the progeny steers were collected for genotyping the SNP. DNA samples were prepared from the materials using DNeasy Blood \& Tissue Kit (QIAGEN, Hilden, Germany).

The BMS, CWT, REA, RT, YE and SFT were measured according to the Japanese meat grading system by certified graders from the Japan Meat Grading Association (Tokyo, Japan) [1]. The predicted breeding values of the BMS, CWT, REA, RT, YE and SFT for the progeny steers were used as the phenotypic values in this study. The breeding values were predicted using carcass records of Japanese Black steers and heifers fattened in the Niigata prefecture, and obtained from the Niigata Prefectural Headquarters, National Federation of Agricultural Cooperative Association (Niigata, Japan).

This study conformed to the guidelines for animal experimentation of the Graduate School of Science and Technology, Niigata University (Niigata, Japan).

\subsection{SNP Genotyping}

The $c .1326 T>G$ SNP was genotyped by the PCR-restriction fragment length polymorphism method as described previously [6]. PCR primers used for PCR-RFLP were 5'-ATTTAGGAAACGACTACTGG-3' and

5'-ATTTGTATTCTCTTATTATCATC-3'. PCR amplifications were performed using $20 \mathrm{ng}$ of the prepared DNA as template in a final volume of $25 \mu \mathrm{l}$ containing $1 \mu \mathrm{M}$ of each primer, $1 \mathrm{U}$ of Mighty Amp DNA polymerase (TaKaRa), and 2 X Mighty Amp buffer (800 $\mu \mathrm{M}$ of each dNTP) (TaKaRa). The PCR conditions were 
carried out as follows: $94^{\circ} \mathrm{C}$ for $4 \mathrm{~min}, 40$ cycles of $94^{\circ} \mathrm{C}$ for $30 \mathrm{~s}$, the annealing temperature of $52^{\circ} \mathrm{C}$ for $30 \mathrm{~s}$, and $72^{\circ} \mathrm{C}$ for $30 \mathrm{~s}$, followed by a further 5 min extension at $72^{\circ} \mathrm{C}$. An aliquot of PCR-amplified products was digested at $65^{\circ} \mathrm{C}$ for $1 \mathrm{~h}$ with restriction enzyme Tsp509I, and electrophoresed on a $2.0 \%$ agarose gel. Agarose gels were stained with ethidium bromide and photographed under an ultraviolet light.

Using this method, 133-bp PCR fragments containing the SNP site were amplified and Tsp509I-digested into 70- and 63-bp fragments at the $T$ allele, but not the $G$ allele: the $G G$ homozygotes, the $T T$ homozygotes and the GT heterozygotes yielded 1 band (133 bp), 2 bands (70 and $63 \mathrm{bp}$ ) and 3 bands (133, 70, and 63 bp), respectively.

\subsection{Statistical Analyses}

Departures from the Hardy-Weinberg equilibrium were tested for the SNP by chi-square test. Statistical comparisons between the allele frequencies at the SNP in the half-sib progeny steers or estimated by maternal alleles in the progeny steers in the Niigata prefecture population and the previous date obtained in Japanese Black cattle population of the Kagoshima prefecture [7] were also performed by chi-square test. The effect of genotypes at the SNP on the predicted breeding values for the BMS, SFT, CWT, REA, RT and YE was analyzed with the model that included the SNP genotype as the fixed effect and the sire as the random effect. Statistical analysis was performed by the MIXED procedures of the SAS program (SAS Institute, Inc., Cary, NC).

\section{Results and Discussion}

Statistically significant differences in the growth-related traits, CWT, REA and RT were detected among the genotypes of the $c .1326 T>G$ SNP in the Niigata prefecture population, with the $G$ allele being associated with a favorable phenotype of these traits by analysis using the model that included the SNP genotype as the fixed effect and the sire as the random effect (Table 1). These results of CWT and REA were consistent with data obtained in previous study in the Kagoshima prefecture population [7], and thus confirmed that the NCAPGC. $1326 T>G$ SNP is a causative variation for bovine CWT [2].

However, no effects of the $c .1326 T>G$ SNP were detected on the SFT and YE (Table 1). Our present study might not have sufficient power to detect the association of the $c .1326 T>G$ SNP with the SFT and YE in the Niigata prefecture population, because of the larger number of segregating QTL for the growth-related trait in the Niigata prefecture population as compared with that in the Kagoshima prefecture population. Further study using a larger number of samples will be needed for the Niigata prefecture population. In addition, this study is the first report to show that the $c .1326 T>G$ SNP is associated with BMS in Japanese Black beef cattle with the $G$ allele being associated with a high level of BMS. Therefore, we concluded that the $c .1326 T>G$ SNP in the NCAPG is useful for effective marker-assisted selection to increase meat quality and meat productivity in Japanese Black beef cattle of the Niigata prefecture.

Table 1. Effects of the $c .1326 T>G$ SNP genotypes on BMS, SFT, CWT, REA, RT and YE in Japanese Black cattle population of the Niigata prefecture.

\begin{tabular}{|c|c|c|c|c|}
\hline \multirow{2}{*}{ Trait $^{1}$} & \multirow{2}{*}{$P$-value } & \multicolumn{3}{|c|}{ Genotype $^{2}$} \\
\hline & & GG & $G T$ & $T T$ \\
\hline BMS & 0.0298 & $1.60^{\mathrm{a}} \pm 0.13$ & $1.45^{\mathrm{a}} \pm 0.05$ & $1.31^{b} \pm 0.04$ \\
\hline SFT & 0.8168 & $-0.12 \pm 0.11$ & $-0.10 \pm 0.04$ & $-0.14 \pm 0.04$ \\
\hline CWT & $<0.0001$ & $78.73^{\mathrm{a}} \pm 8.87$ & $59.58^{\mathrm{b}} \pm 3.44$ & $33.19^{c} \pm 2.97$ \\
\hline REA & 0.0009 & $8.09^{\mathrm{a}} \pm 1.24$ & $8.18^{a} \pm 0.48$ & $5.81^{b} \pm 0.42$ \\
\hline $\mathrm{RT}$ & 0.0013 & $0.77^{\mathrm{a}} \pm 0.10$ & $0.70^{\mathrm{a}} \pm 0.04$ & $0.53^{b} \pm 0.03$ \\
\hline YE & 0.4960 & $0.85 \pm 0.24$ & $1.07 \pm 0.09$ & $0.94 \pm 0.08$ \\
\hline
\end{tabular}

${ }^{1}$ BMS, Beef marbling score (unit); SFT, Subcutaneous fat thickness (cm); CWT, Carcass weight (kg); REA, Rib eye area (cm²); RT, Rib thickness $(\mathrm{cm})$; YE, Yield estimate $(\%) .{ }^{2}$ The breeding values are given as least squares means \pm SE. The least squares means and SE are calculated by the MIXED procedures of the SAS program (SAS Institute, Inc., Cary, NC). ${ }^{\mathrm{a}, \mathrm{b}, \mathrm{c}}$ Mean values at different genotypes without a common superscript letter significantly differ $(P<0.001)$. 
Table 2. Frequencies of the $c .1326 T>G$ SNP genotypes in Japanese Black cattle population of the Niigata prefecture.

\begin{tabular}{ccc}
\hline Genotype & No. of animals & Frequency \\
\hline$G G$ & 9 & 0.060 \\
$G T$ & 60 & 0.403 \\
$T T$ & 80 & 0.537 \\
\hline
\end{tabular}

Genotyping the 149 paternal half-sibprogeny steers for the c.1326T $>G$ SNP revealed 9 animals homozygous for the $G$ allele, 60 animals heterozygous for the $G$ allele and the $T$ allele and 80 animals homozygous for the $T$ allele (Table 2). The observed frequencies of the SNP genotypes in the Niigata population of Japanese Black cattle are shown in Table 2. The observed and expected heterozygosity values at the SNP conformed to the Hardy-Weinberg equilibrium in the Niigata prefecture population. Statistically significant difference was detected between the allele frequencies obtained in the half-sib progeny steers in the Niigata prefecture population and obtained in Japanese Black cattle population of the Kagoshima prefecture [7]. The frequency of the favorable $G$ allele of the $c .1326 T>G$ SNP in the Niigata prefecture population was lower than that of the Kagoshima prefecture population. However, no statistically significant difference was detected between the allele frequencies estimated by maternal alleles in the half-sib progeny steers in the Niigata prefecture population and obtained in Japanese Black cattle population of the Kagoshima prefecture [7] (data not shown).

Many QTLs for the growth-related trait are thought to be fixed for favorable alleles in the Kagoshima prefecture population [8]. We should note that the female population in Japanese Black cattle of the Niigata prefecture possesses the favorable allele ( $G$ allele) frequency at the $c .1326 T>G$ SNP, which is comparable to the data observed in the Kagoshima prefecture population [7].

\section{Conclusion}

In this study, we showed the replicated and novel association of the $c .1326 T>G$ SNP with the CWT and REA, and the BMS, respectively. Therefore, we concluded that the $c .1326 T>G$ SNP in the NCAPG is useful for effective marker-assisted selection to increase meat quality and meat productivity in Japanese Black beef cattle of the Niigata prefecture. Furthermore, it is important to note that the female population in Japanese Black cattle of the Niigata prefecture possesses the favorable allele ( $G$ allele) frequency at $c .1326 T>G$, which is comparable to the data observed in the Kagoshima prefecture population. These results suggest that many QTLs for the growthrelated trait may be fixed for favorable alleles in the female population in Japanese Black cattle of the Niigata prefecture, and that a selection for high growth performance in sire's side in Japanese Black cattle of the Niigata prefecture will be useful.

\section{Acknowledgements}

This work was supported by a Grant-in-Aid for Scientific Research (B) (no. 14360166) from the Ministry of Education, Culture, Sports, Science and Technology of Japan, and by the research funds of Japanese Livestock Technology Association.

\section{References}

[1] JMGA (1988) New Beef Carcass Grading Standards. Japan Meat Grading Association, Tokyo, Japan.

[2] Setoguchi, K., Furuta, M., Hirano, T., Nagao, T., Watanabe, T., Sugimoto, Y. and Takasuga, A. (2009) Cross-Breed Comparisons Identified a Critical 591 kb Region for Bovine Carcass Weight QTL (CW-2) on Chromosome 6 and the Ile-442-Met Substitution in NCAPG as a Positional Candidate. BMC Genetics, 10, 43. http://dx.doi.org/10.1186/1471-2156-10-43

[3] Eberlein, A., Takasuga, A., Setoguchi, K., Pfuhl, R., Flisikowski, K., Fries, R., Klopp, N., Fürbass, R., Weikard, R. and Kühn, C. (2009) Dissection of Genetic Factors Modulating Fetal Growth in Cattle Indicates a Substantial Role of the Non-SMC Condensing I Complex, Subunit G (NCAPG) Gene. Genetics, 183, 951-964. http://dx.doi.org/10.1534/genetics.109.106476

[4] Karim, L., Takeda, H., Lin, L., Druet, T., Arias, J.A., Baurain, D., Cambisano, N., Davis, S.R., Farnir, F., Grisart, B., Harris, B.L., Keehan, M.D., Littlejohn, M.D., Spelman, R.J., Georges, M. and Coppieters, W. (2011) Variants Modulating the Expression of a Chromosome Domain Encompassing PLAG1 Influence Bovine Stature. Nature Genetics, 
43, 405-413. http://dx.doi.org/10.1038/ng.814

[5] Weikard, R., Altmaier, E., Suhre, K., Weinberger, K.M., Hammon, H.M., Albrecht, E., Setoguchi, K., Takasuga, A. and Kuehn, C. (2010) Metabolomic Profiles Indicate Distinct Physiological Pathways Affected by Two Loci with Major Divergent Effect on Bostaurus Growth and Lipid Deposition. Physiological Genomics, 42A, 79-88. http://dx.doi.org/10.1152/physiolgenomics.00120.2010

[6] Setoguchi, K., Watanabe, T., Weikard, R., Albrecht, E., Kühn, C., Kinoshita, A., Sugimoto, Y. and Takasuga, A. (2011) The SNP c.1326T>G in the Non-SMC Condensin I Complex, Subunit G (NCAPG) Gene Encoding a p.Ile442Met Variant Is Associated with An increase in Body Frame Size at Puberty in Cattle. Animal Genetics, 42, 650-655. http://dx.doi.org/10.1111/j.1365-2052.2011.02196.x

[7] Hoshiba, H., Setoguchi, K., Watababe, T., Kinoshita, A., Mizoshita, K., Sugimoto, Y. and Takasuga, A. (2013) Comparison of the Effects Explained by Variations in the Bovine PLAG1 and NCAPG Genes on Daily Body Weight Gain, Linear Skeletal Measurements and Carcass Traits in Japanese Black Steers from a Progeny Testing Program. Animal Science Journal, 84, 529-534. http://dx.doi.org/10.1111/asj.12033

[8] Sukegawa, S., Miyake, T., Takahagi, Y., Murakami, H., Morimatsu, F., Yamada, T. and Sasaki, Y. (2010) Replicated Association of the Single Nucleotide Polymorphism in EDG1 with Marbling in Three General Populations of Japanese Black Beef Cattle. BMC Research Notes, 3, 66-68. http://dx.doi.org/10.1186/1756-0500-3-66

\section{Submit or recommend next manuscript to SCIRP and we will provide best service for you:}

Accepting pre-submission inquiries through Email, Facebook, Linkedin, Twitter, etc A wide selection of journals (inclusive of 9 subjects, more than 200 journals)

Providing a 24-hour high-quality service

User-friendly online submission system

Fair and swift peer-review system

Efficient typesetting and proofreading procedure

Display of the result of downloads and visits, as well as the number of cited articles

Maximum dissemination of your research work

Submit your manuscript at: http://papersubmission.scirp.org/ 